\title{
A Neural Approach for Reliable and Fault Tolerant Wireless Sensor Networks
}

\author{
Vijay Kumar (Member, IEEE) ${ }^{1}$ \\ Department of Computer \\ Engineering, \\ M. M. University, Mullana, \\ Ambala, Haryana, India- 133207
}

\author{
R. B. Patel (Member, IEEE) ${ }^{2}$ \\ Department of Computer \\ Engineering, \\ M. M. University, Mullana, \\ Ambala, Haryana, India- 133207
}

\author{
Manpreet Singh (Member,IEEE) ${ }^{3}$ \\ Department of Computer \\ Engineering, \\ M. M. University, Mullana, \\ Ambala, Haryana, India- 133207
}

\author{
Rohit Vaid ${ }^{4}$ \\ Department of Computer \\ Engineering, \\ M. M. University, Mullana, \\ Ambala, Haryana, India- 133207
}

\begin{abstract}
This paper presents a neural model for reliable and fault tolerant transmission in Wireless Sensor Networks based on Bi-directional Associative Memory. The proposed model is an attempt to enhance the performances of both the cooperative and non cooperative Automatic Repeat Request (ARQ) schemes in terms of reliability and fault tolerance. We have also demonstrated the performances of both the schemes with the help of suitable examples.
\end{abstract}

Keywords- Reliability; Fault tolerance; Bi-directional Associative Memory; Wireless Sensor Network.

\section{INTRODUCTION}

Wireless sensor networks (WSNs) [9][2] are the topic of intense academic and industrial studies. Research is mainly focused on energy saving schemes to increase the lifetime of these networks [4][5]. There is an exciting new wave in sensor applications-wireless sensor networking- which enables sensors and actuators to be deployed independent of costs and physical constraints of wiring. For a wireless sensor network to deliver real world benefits, it must support the following requirements in deployment: scalability, reliability, responsiveness, power efficiency and mobility.

The complex inter-relationships between these characteristics are a balance; if they are not managed properly, the network can suffer from overhead that negates its applicability. In order to ensure that the network supports the application's requirements, it is important to understand how each of these characteristics affects the reliability.

\section{A. Scalability and Reliability}

Network reliability and scalability are closely coupled and typically they act against each other. In other words, it is very difficult to build a reliable ad hoc network as the number of nodes increases [7]. This is due to network overhead that comes with increased size of network. In ad hoc network, there is no predefined topology or shape. Therefore, any node wishing to communicate with other nodes should generate more control packets than data packets. Moreover, as network size increases, there is more risk that communication links get broken, which will end up with creating more control packets. In summary, more overhead is unavoidable in a larger scale wireless sensor network to keep the communication path intact.

\section{B. Reliability and power efficiency}

Power efficiency also plays a very important role in this complex equation. To design a low power wireless sensor network, the duty cycle of each node needs to be reduced. The drawback is that as the node stays longer in sleep mode [3] to save the power, there is less probability that the node can communicate with its neighbors and may also lower the reliability due to lack of exchange of control packets and delays in the packet delivery.

\section{Reliability and responsiveness}

Ability of the network to adapt quickly the changes in the topology is known as responsiveness. For better responsiveness, there should be more issue and exchange of control packets in ad hoc network, which will naturally result in less reliability.

\section{Mobility and reliability}

A wireless sensor network that includes a number of mobile nodes should have high responsiveness to deal with the mobility. The mobility effect on responsiveness will compound the reliability challenge.

Many applications for wireless sensor networks require immediate and guaranteed action; for example medical emergency alarm, fire alarm detection, instruction detection [6]. In these situations packets has to be transported in a reliable way and in time through the sensor network. Thus, besides the energy consumption, delay and data reliability becomes very relevant for the proper functioning of the network.

Direct communication between any node and sink could be subject only to just a small delay, if the distance between the source and the destination is short, but it suffers an important energy wasting when the distance increases. Therefore often mutihop short range communications through other sensor nodes, acting as intermediate relay, are preferred in order to reduce the energy consumption in the network. In such a scenario it is necessary to define efficient technique that can ensure reliable communication with very tight delay constraint. In this work we focus attention on the control of data and reliability in multihop scenario.

A simple implementation of ARQ is represented by the Stop and Wait technique that consists in waiting the acknowledgement of each transmitted packet before 
transmitting the next one, and retransmit the same packet in case it is lost or wrongly, received by destination [8].

We extend here this analysis by introducing the investigation of the delay required by the reliable data delivery task. To this aim we investigate the delay required by a cooperative ARQ mechanism to correctly deliver a packet through a multihop linear path from a source node to the sink. In particular we analyze the delay and the coverage range of the nodes in the path, therefore the relation between delay and the number of cooperative relays included in the forwarding process.

\section{NEURAL NETWORK IN WSN}

Recently there is a strong interest to use neural network in energy efficient methods of WSNs. Algorithm developed in artificial neural network can be easily developed to wireless sensor network platforms and can satisfy the requirement for sensor networks kike: simple parallel distributed computation distributed storage, data robustness \& auto classification of sensor readings, fault tolerance \& low computation. Neural networks can help through dimensionality reduction obtain from the outputs of the neural networks clustering algorithms, leads to lower communication cost \& energy saving. The other reason to use neural network based methods in WSNs is the analogy between WSNs \& ANNs. As authors [1] strongly believe that ANNs exhibit exactly the same architecture WSNs since neural networks compared to sensor nodes \& communications corresponds to radio links classification techniques.

Cluster based routing are most frequently used energy efficient routing protocols in WSNs which avoid single gateway architecture through developing of network into several clusters, while cluster head of each cluster play the role of a local base station.

Sensor node spends maximum energy in data communication by radio unit. Data communication in WSN must be reliable \& must consume maximum power. Every sensor node consists of multiple sensors embedded in the node $\&$ this is a source of data. Rather communicating these data streams directly to the neighbouring node or base station, these data streams used to be classified.

\section{PERFORMANCE OF COOPERATIVE ARQ USING MARKOV MODEL}

Fig. 1 shows the network structure with linear multihop path consist of source node (node $\mathrm{n}=1$ ), destination (node $\mathrm{n}=$ $\mathrm{N}$ ) and $(\mathrm{N}-2) * t$ intermediate relay nodes deployed at equal distance where $t$ is the number of parallel path of intermediate relay nodes between source and destination. Each path is composed by $\mathrm{Z}=\mathrm{N}-1$ links. Suppose that all the nodes have circular radio coverage with the same transmission range $R_{t}$. When a sensor transmits a packet, it is received by all the sensors in a listen state inside the coverage area of the sender.

When a packet is transmitted, it can be forwarded towards the destination by only those nodes which are closer to the destination than the transmitter.

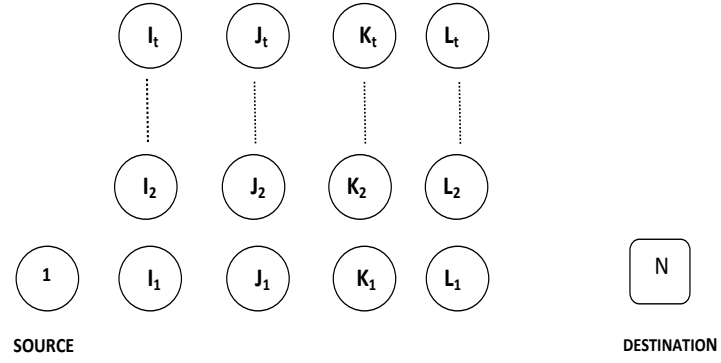

Fig.1 Network structure with Linear multihop path

\section{A. Discrete Parameter MARKOV CHAIN with Absorbing State}

Packet transfer from source to destination via intermediate forwarders can be treated as a state diagram of discrete parameter Markov chain with absorbing state. An absorbing state is a state from which there is zero probability of exiting. An absorbing Markov system is a Markov system that contains at least one absorbing state, and is such that it is possible to get from each non absorbing state to some absorbing state in one or more time steps. Consider $\mathrm{p}$ be the probability of successful transmission of a packet to an intermediate relay node inside the coverage range. Therefore $1-p$ will be the probability of unsuccessful transmission of packet.

For each node $n$, the probability to correctly deliver a packet to a node that is $R_{t}$ links distant is equal to $p$. So the probability that the packet is not correctly received will be $(1-p)$, while it is correctly received from the immediately previous node with a probability $\mathrm{p}$. Therefore the packet will be forwarded by the previous node with a probability $(1-p) p$. If this node also has not correctly received the packet send by node $\mathrm{n}$ then the packet will be forwarded by the node previous to previous with a probability $(1-\mathrm{p})^{2} \mathrm{p}$. If none of the node in the coverage area of the transmitter receives a correct packet it is necessary to ask the retransmission of the packet by the source node. It is possible to describe the process concerning one data packet forwarding from the source node $n=1$ to the destination $\mathrm{n}=\mathrm{N}$ with a discrete time Markov chain with absorbing state. Packet transmitted by a node will be further forwarded by a node in the coverage range of the transmitter which is furthest node from the source and has correctly received the packet.

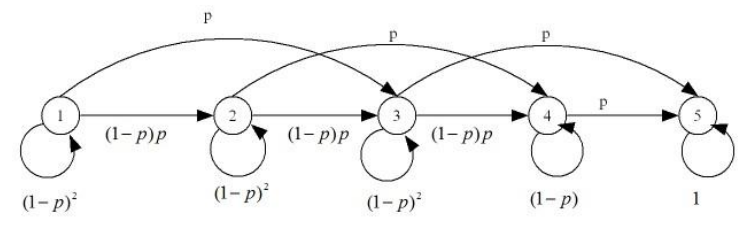

Fig. 2 Packet transmission in Cooperative ARQ

Consider a single multihop linear path consisting five sensors with four links as shown in Fig. 2. Assume transmission range of each sensor node is $R_{t}=2$ unit. State transition probability matrix for a successful transmission of a packet under cooperative automatic repeat request will be as under: 


$$
P_{\text {Success }}=\left[\begin{array}{llccc}
(1-p)^{2} & p(1-p) & p & 0 & 0 \\
0 & (1-p)^{2} & p(1-p) & p & 0 \\
0 & 0 & (1-p)^{2} & p(1-p) & p \\
0 & 0 & 0 & (1-p) & p \\
0 & 0 & 0 & 0 & 1
\end{array}\right]
$$

Similarly we can find the probability matrix for link error by replacing (1-p) with $q$. In fig. 2 states 1 through 4 are transient state while state 5 is an absorbing state.

In general, we consider a Markov chain with $n$ states, $\mathrm{s}_{1}, \mathrm{~s}_{2}$, $\ldots, \mathrm{s}_{\mathrm{n}} . \mathrm{S}_{\mathrm{n}}$ will be the absorbing state, and the remaining state will be transient. The transition probability matrix of such a chain may be partitioned so that

$$
P=\left[--\frac{Q}{0}--\mid--\frac{C}{1}--\right]
$$

Where $Q$ is an (n-1) by (n-1) substochastic matrix, describing the probabilities of transition only among the transient states. $\mathrm{C}$ is a column vector and 0 is a row vector of (n-1) zeros. Now the k-step transition probability matrix $\mathrm{P}^{\mathrm{k}}$ has the form

$$
P^{k}=\left[--\frac{Q^{k}}{0}--\mid--\frac{C^{\prime}}{1}--\right]
$$

Where $C^{\prime}$ is a column vector whose elements will be of no further use and hence need not be computed. The (i, j) entry of matrix $Q^{k}$ denotes the probability of arriving in (transient) state $\mathrm{s}^{\mathrm{j}}$ after exactly $\mathrm{k}$ steps starting from (transient) state $\mathrm{s}^{\mathrm{i}}$. It can be shown that $\sum_{k=0}^{t} Q^{k}$ converges as t approaches infinity. This imply that the inverse matrix $(\mathrm{I}-\mathrm{Q})^{-1}$, called the fundamental matrix, $\mathrm{M}$, exists and is given by

$$
M=(I-Q)^{-1}=I+Q+Q^{2}+\ldots=\sum_{k=0}^{\infty} Q^{k} \quad . \quad \text { The }
$$

fundamental matrix is used for calculating the expected no. of steps to absorption. The number of times, starting in state $i$, and expected to visit state $\mathrm{j}$ before absorption is the $\mathrm{ij}^{\text {th }}$ entry of $\mathrm{M}$. The total no. of steps expected before absorption equals the total no. of visits expected to make to all the non absorption states. This is the sum of all the entries in the $\mathrm{i}^{\text {th }}$ row of $\mathrm{M}$.

Suppose $\mathrm{p}=0.8$, then $\mathrm{Q}$ will be as under

$$
Q_{\text {Success }}=\left[\begin{array}{lccc}
.04 & .16 & .8 & 0 \\
0 & .04 & .16 & .8 \\
0 & 0 & .04 & .16 \\
0 & 0 & 0 & .2
\end{array}\right]
$$

Therefore fundamental matrix $M=(I-Q)^{-1}$

$=\left[\begin{array}{llll}25 / 24 & 25 / 144 & 775 / 864 & 305 / 864 \\ 0 & 25 / 24 & 25 / 144 & 155 / 144 \\ 0 & 0 & 25 / 24 & 5 / 24 \\ 0 & 0 & 0 & 5 / 4\end{array}\right]$

Thus the states $1,2,3$ and 4 are respectively executed $25 / 24,25 / 144,775 / 864,305 / 864$ times on the average. If $t_{1}, t_{2}$, $t_{3}$ and $t_{4}$ respectively is the time for one time execution of the states 1, 2, 3 and 4 then total time required to transmit a packet from source node 1 to destination node 5 is equal to :

$\mathrm{T}=25 / 24 \mathrm{t}_{1}+25 / 144 \mathrm{t}_{2}+775 / 864 \mathrm{t}_{3}+305 / 864 \mathrm{t}_{4}$ unit times.

If $\mathrm{t}_{1}=\mathrm{t}_{2}=\mathrm{t}_{3}=\mathrm{t}_{4}=\mathrm{t}$ then $\mathrm{T}=2.4645$ unit times.

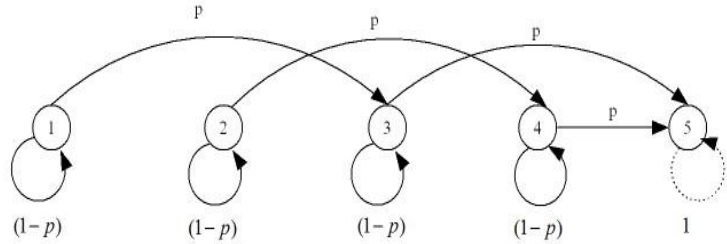

Fig 3. Packet transmission in Non-Cooperative ARQ as a discrete parameter Markov Chain with absorbing state

In non-Cooperative $\mathrm{ARQ}$, a packet transmitted by source node is received by a node at distance $R_{t}$ towards the destination from source and is forwarded by the node if packet received correctly otherwise transmitter is forced for retransmission. Other intermediate nodes between the transmitter and the node at distance $R_{t}$ remains in sleep mode as they will never be involved in packet forwarding process. State transition probability matrix for successful transmission of the packet for non-cooperative ARQ will be as under:

$$
P_{\text {Success }}=\left[\begin{array}{lllll}
1-p & 0 & p & 0 & 0 \\
0 & 1-p & 0 & p & 0 \\
0 & 0 & 1-p & 0 & p \\
0 & 0 & 0 & (1-p) & p \\
0 & 0 & 0 & 0 & 1
\end{array}\right]
$$

Suppose $\mathrm{p}=0.8$, then $\mathrm{Q}$ will be as under

$Q_{\text {Success }}=\left[\begin{array}{rrrr}.2 & 0 & .8 & 0 \\ 0 & .2 & 0 & .8 \\ 0 & 0 & .2 & 0 \\ 0 & 0 & 0 & 0\end{array}\right]$

Therefore fundamental matrix

$$
M=(I-Q)^{-1}=\left[\begin{array}{llll}
5 / 4 & 0 & 5 / 4 & 0 \\
0 & 5 / 4 & 0 & 1 \\
0 & 0 & 5 / 4 & 0 \\
0 & 0 & 0 & 1
\end{array}\right]
$$

Thus the states $1,2,3$ and 4 are respectively executed 5/4, $0,5 / 4,0$ times on the average if source node is considered as node 1 . If $t_{1}, t_{2}, t_{3}$ and $t_{4}$ respectively is the time for one time execution of the states $1,2,3$ and 4 then total time required to transmit a packet from source node 1 to destination node 5 is equal to:

$\mathrm{T}=5 / 4 \mathrm{t}_{1}+5 / 4 \mathrm{t}_{3}$ units times.

If $\mathrm{t}_{1}=\mathrm{t}_{2}=\mathrm{t}_{3}=\mathrm{t}_{4}=\mathrm{t}$ then $\mathrm{T}=2.5$ unit times. 
It has been observed that packet delivery is more reliable and timely in case of cooperative ARQ, where as non cooperative ARQ is better in terms of power efficiency of sensor nodes as most of the sensors do not participate in packet forwarding process.

\section{SySTEM MODEl FOR FAULT TOLERANT AND RELIABLE TRANSMISSION}

In Co-operative ARQ scheme, a packet may not be successfully transmitted to the destination because of the following reasons; 1 . Probable forwarders inside the coverage are in good working condition but have not received the packet correctly because of noise etc. and 2. All the forwarders are in failure condition.

Our proposed model is an attempt to still improve the fault tolerance \& reliability of packet delivery process in WSN. This model addresses both the reasons of unsuccessful packet delivery in co-operative ARQ scheme. Proposed model is most suitable for those networks where the events that can occur in the network are from a predefined set of events. Assume that sensor node has to sense a packet from a set $\mathrm{P}=\left\{\mathrm{p}_{1}, \mathrm{p}_{2}, \mathrm{p}_{3}, \mathrm{p}_{4}, \mathrm{p}_{5}, \ldots, \mathrm{p}_{10}\right\}$ where the size of each packet is $\mathrm{k}$-bit. Larger is the size of a packet, more will be the chances of faulty delivery of packet to the next probable forwarder/destination because of noise etc. Two possible solutions for reliable packet delivery at the next probable forwarder/destination are as under-

1. Data compression before transmission at the source for reducing the size of the packet to minimize the chances of faulty packet delivery at next probable forwarder/destination.

If we compress the packet using ART1network, the packet needs to be splitted into small frames. These frames are fed into an ART1 network for self organization, and the output represents the class indices for these frames. After training, these indices and their corresponding prototypes are transmitted to the destination. The packet is then reconstructed according to these indices and prototypes. The benefit from this operation is measured by the compression ratio $(\mathrm{Q})$, which is computed as follows: $\mathrm{Q}=\mathrm{NF} /\left(\mathrm{C} \cdot \mathrm{N}+\mathrm{F} \cdot \log _{2} \mathrm{C}\right)$.

Where $\mathrm{N}$ is the dimension of the frames, $\mathrm{F}$ is the total number of frames and $\mathrm{C}$ is the total number of classes formed during learning.

On the other hand, the price we pay is measured by the distortion ratio defined as:

$$
E=\sum_{j=1}^{F} \sum_{i=1}^{N}\left(I_{i j}-I_{i j}^{\prime}\right)^{2} \text { Where } \mathrm{I}_{\mathrm{ij}} \text { is the value in original }
$$

packet and $I_{i j}^{\prime}$ is the corresponding value in reconstructed packet. In packet compression, vigilance parameter is used to control the trade-off between $\mathrm{Q}$ and $\mathrm{E}$.

2. Transmitting a small size packet (vector) associated to the original packet of very large size after encoding the associations between these hetro-associative vectors. Our proposed model is based on neural network concept.
Transmitting a associated vector of small size has better reliability \& fault tolerance over a compressed packet.

Discrete Bi-directional Associative Memory (BAM) neural network is used for encoding the associations. Bi-directional associative memory is a hetro associative recurrent neural network consisting of two layers as shown in Fig. 4. Layer-1 has $\mathrm{n}$ units and layer-2 has $\mathrm{m}$ units. Synaptic strength of various connections from the layer- 1 to layer- 2 is represented by the weight matrix $\mathrm{W}_{\mathrm{nXm}}$ and the weight matrix from layer- 2 to layer-1 is $W_{n x m}^{T}$. This is a fully connected network, wherein the inputs are different from the outputs. After training the network, if vector $\mathrm{A}$ is presented over the network then it recalls the associated vector $\mathrm{B}$ and similarly vector $\mathrm{B}$ recalls vector $\mathrm{A}$ if vector $\mathrm{B}$ is presented over the transpose weight matrix $W^{T}$.

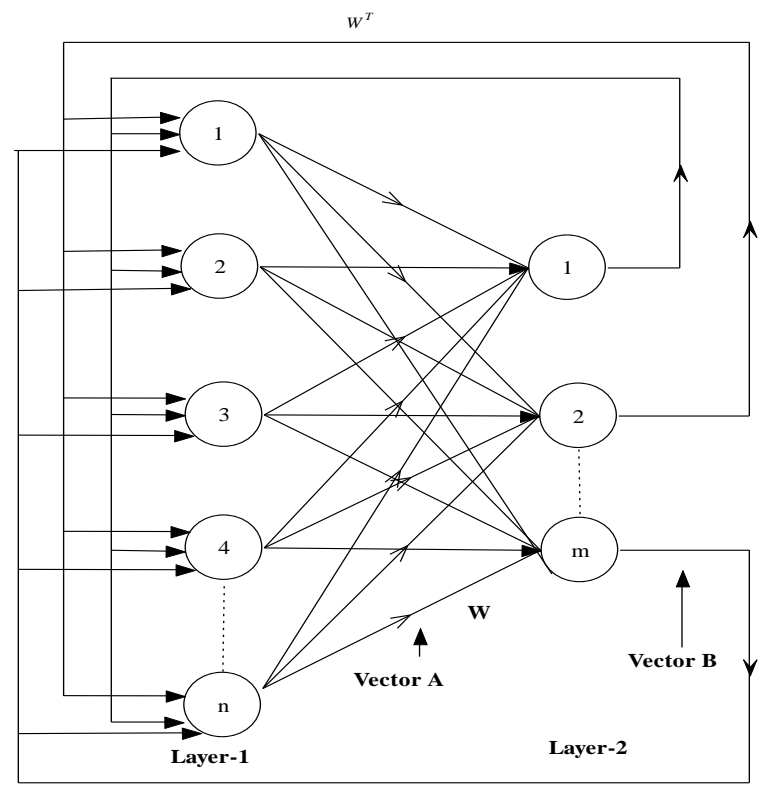

Fig4. Architecture of Bi-directional associative memory

Training procedure (Encoding the associations):

Suppose we want to encode the association between the hetro-associative patterns for the given problem space.

\begin{tabular}{|c|c|}
\hline $\begin{array}{c}\text { Vector A } \\
\text { (Actual packet of size } n)\end{array}$ & $\begin{array}{c}\text { Associated Vector } \\
\mathrm{B}\end{array}$ \\
\hline Training pair 1: $A_{1}$ & $B_{1}$ \\
\hline Training pair 2: $A_{2}$ & $B_{2}$ \\
$\vdots$ & $\vdots$ \\
\hline Training pair $\mathrm{A} A_{k}$ & $B_{k}$ \\
\hline
\end{tabular}

Binary Version

\begin{tabular}{|c|c|}
\hline $\begin{array}{c}\text { Vector } A \\
\text { Actual packet of size } n)\end{array}$ & $\begin{array}{c}\text { Associated Vector } \\
\mathrm{B}\end{array}$ \\
\hline Training pair $1: A_{1}^{\prime}$ & $B_{1}^{\prime}$ \\
\hline Training pair 2: $A_{2}^{\prime}$ & $B_{2}^{\prime}$ \\
\hline Training pair $\mathrm{k}: A_{k}^{\prime}$ & $B_{k}^{\prime}$ \\
\hline
\end{tabular}

Bipolar Version
After the training, weight matrix $W=\sum_{i=1}^{k}\left(A_{i}^{\prime}\right)^{T} B_{i}^{\prime}$.

In this proposed model BAM is embedded in every source node and destination node. At all the source nodes, before transmitting a packet $\mathrm{A}$, it is presented over the BAM network 
through weight matrix $\mathrm{W}$ to get a smaller associated vector $\mathrm{B}_{\mathrm{i}}$. Associated vector $B_{i}$ is transmitted and more successfully delivered at the probable next forwarder/destination, where it is again presented over the embedded BAM network through $\mathrm{W}^{\mathrm{T}}$ to ensure retrieval of the actual packet $\mathrm{A}_{\mathrm{i}}$. If receiver is not the final destination then associated vector $B_{i}$ is further transmitted towards the destination until destination is reached.

Activation function used in BAM is described as:-

Output $=\mathrm{f}(\mathrm{NET})=\left\{\begin{array}{l}1, \text { if NET }>0 \\ 0, \text { if NET }<0 \\ \text { Previous output, if NET }=0\end{array}\right.$

Where NET $= \begin{cases}\text { A.W } & \text { at Layer - } 2 \\ \text { B. } W^{\mathrm{T}} & \text { at Lay er }-1\end{cases}$

The memory capacity of BAM network is defined as min $(n, m)$; where $n$ is the number of units in layer-1 (size of vector A i.e. the original packet) and $\mathrm{m}$ is the number of units in layer-2 (size of vector $B$ i.e. the vector to be transmitted). Size of the associated vector $B_{i}$ corresponding to the original packet $\mathrm{A}_{\mathrm{i}}$ depends on the total number of different packets (events) to encounter in the network irrespective of the size of original packet. Even if the packet size is very large (say $n=10000$ bits) and the total number of different packet to be handled by the network is small (say $\mathrm{K}=10$ ) then associated vector $\mathrm{B}$ to be transmitted should be of the size $\mathrm{m} \leq 10$ bits for the reliable transmission of data. Even if the associated vector B has been received incorrectly at the next forwarder/destination, network may recall the actual packet despite of the corrupted signal received at next forwarded/destination (input signal to the BAM network) as network has the power of generalization. Moreover, BAM net is more fault tolerant with corrupted signal due to "missing" bits than that of "mistaken" bits.

This can be illustrated with the help of example 1:

Example 1:

Original packet

$\mathrm{A}_{1}=\left[\begin{array}{llllllll}1 & 0 & 1 & 0 & 1 & 0 & 1 & 1\end{array}\right]$

$\mathrm{A}_{2}=\left[\begin{array}{llllllll}1 & 1 & 0 & 1 & 1 & 1 & 0 & 1\end{array}\right]$

Associated packet for the transmission

$$
\mathrm{B}_{1}=\left[\begin{array}{lll}
1 & 1 & 1
\end{array}\right]
$$$$
\mathrm{B}_{2}=\left[\begin{array}{lll}
1 & 0 & 0
\end{array}\right]
$$

Bi-Polar versions

$$
\begin{aligned}
& A_{1}^{\prime}=\left[\begin{array}{llllllll}
1 & -1 & 1 & -1 & 1 & -1 & 1 & 1
\end{array}\right] \\
& B_{1}^{\prime}=\left[\begin{array}{lll}
1 & 1 & 1
\end{array}\right] \\
& A_{2}^{\prime}=\left[\begin{array}{llllllll}
1 & 1 & -1 & 1 & 1 & 1 & -1 & 1
\end{array}\right] \\
& B_{2}^{\prime}=\left[\begin{array}{lll}
1 & -1 & -1
\end{array}\right] \\
& \text { Weight matrix } W=\sum_{i=1}^{k}\left(A_{i}^{\prime}\right)^{T} B_{i}^{\prime}
\end{aligned}
$$

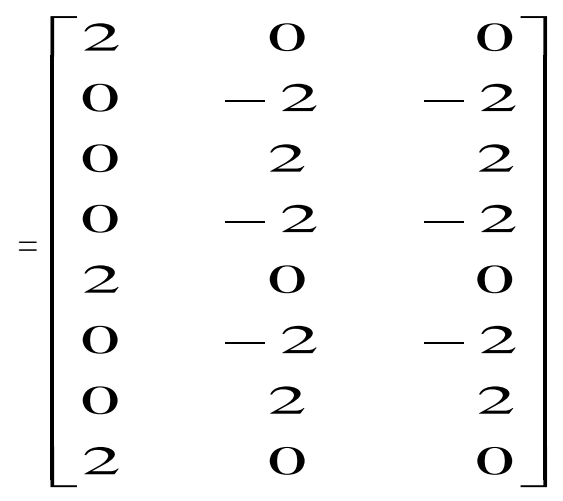

Associated pattern corresponding to packet $A_{1}$ to be transmitted from the source node is computed as:

$$
\begin{aligned}
& N E T=A_{1}^{\prime} \cdot W=\left[\begin{array}{ccc}
6 & 10 & 10
\end{array}\right] \\
& \text { Output=F[NET }]=\left[\begin{array}{ccc}
1 & 1 & 1
\end{array}\right]=\mathrm{B}_{1} .
\end{aligned}
$$

This pattern $\mathrm{B}_{1}$ is transmitted towards the destination and is more reliably received by the next probable forwarder as the size of the pattern $B_{1}$ is smaller than the actual packet $A_{1}$. Received pattern is presented over the BAM network embedded at the next probable forwarder to ensure the correct delivery of the packet. Retrieval of the associated actual packet at the forwarder is shown as under:

$$
\begin{aligned}
\text { NET }=B_{1}^{\prime} \cdot W^{T} & =\left[\begin{array}{rrrrrrrr}
2 & -4 & 4 & -4 & 2 & -4 & 4 & 2
\end{array}\right] \\
\text { Output } & =\left[\begin{array}{llllllll}
1 & 0 & 1 & 0 & 1 & 0 & 1 & 1
\end{array}\right]=A_{1}
\end{aligned}
$$

In case forwarder/Destination receives the corrupted signal, BAM network has the power of generalization to recall the actual associated packet within its fault tolerance limit. This is illustrated in the example2.

\section{Example 2:}

Suppose signal received is faulty at the second missing bit i.e. $B_{1}=\left[\begin{array}{lll}1 & 0 & 1\end{array}\right]$. The packet retrieval by the destination is shown as under

$$
\begin{aligned}
\text { NET }=B_{1}^{\prime} \cdot W^{T} & =\left[\begin{array}{rrrrrrrr}
2 & -2 & 2 & -2 & 2 & -2 & 2 & 2
\end{array}\right] \\
\text { Output } & =\left[\begin{array}{llllllll}
1 & 0 & 1 & 0 & 1 & 0 & 1 & 1
\end{array}\right]=A_{1}
\end{aligned}
$$

This shows that the packet has been retrieved correctly at the destination despite of corrupted signal. Similarly for fault at third missing bit i.e. $B_{1}=\left[\begin{array}{lll}1 & 1 & 0\end{array}\right]$.

$$
\begin{aligned}
\text { NET }= & B_{1}^{\prime} \cdot W^{T}=\left[\begin{array}{rrrrrrrr}
2 & -2 & 2 & -2 & 2 & -2 & 2 & 2
\end{array}\right] \\
\text { Output } & =\left[\begin{array}{llllllll}
1 & 0 & 1 & 0 & 1 & 0 & 1
\end{array}\right]=A_{1}
\end{aligned}
$$




\section{CONCLUSION}

In this work we have presented the neural approach to analyze the performance of cooperative and non cooperative ARQ schemes in terms of delay and power efficiency. This model explores the advantages of both the schemes. With the embedded BAM at each sensor, packet delivery is more reliable and timely than that of the normal cases of cooperative and non cooperative ARQ as BAM is capable to retrieve the actual packet despite of corrupted associated pattern delivery at the forwarder within its fault tolerance limit.

\section{FUTURE SCOPE}

Fault tolerance power of the proposed model may still be improved by cascading BAM structure with a recurrent network in asynchronous mode. Corrupted input pattern received at the next forwarder may be recovered partially by recurrent network bit by bit before directly applying to the BAM structure. A classification based neural network may also be used to further improve the efficiency of the proposed model.

\section{REFERENCES}

[1] Oldewurtel, Frank and Mahonen, Petri, (2006), "Neural Wireless Sensor Networks", International Conference on systems \& Networks Communications, ICSNS'06, pp. 28-28 .

[2] Tubaishat $M$ \& Madria S, "Sensor networks: an overview" IEEE Potentials Volume 22, Issue 2, April- May 2003 pp: 20-23, 2003.

[3] C. F. Chiasserini and M. Garetto, "An analytical model for wireless sensor networks with sleeping nodes", IEEE Trans. Mobile Computing, vol. 5, no. 12, pp: 1706-1718, 2006.

[4] Pal, Y., Awasthi, L.K., Singh, A.J., "Maximize the Lifetime of Object Tracking Sensor Network with Node-to-Node Activation Scheme", in Proceeding of Advance Computing Conference, pp: 1200 - 1205, 2009.

[5] Yan-liang Jin, Hao-jie Lin, Zhu-ming Zhang, Zhen Zhang, Xu-yuan Zhang, "Estimating the Reliability and Lifetime of Wireless Sensor Network", in Proceeding of Wireless Communications, Networking and Mobile Computing (WiCOM 2008), pp: 2181 -2186, 2008.

[6] L. Bernardo, R. Oliveria, R. Tiago, P. Pinto, "A Fire Monitoring Application for Scattered Wireless Sensor Network", in the proceeding of WinSys 2007, on July 28-31, 2007.

[7] Wenyu Cai, Xinyu Jin, Yu Zhang, Kangsheng Chen, Jun Tang, "Research on Reliability Model of Large-Scale Wireless Sensor Networks", in Proceeding of Wireless Communications, Networking and Mobile Computing (WiCOM 2006) pp: 1-4, 2006.

[8] AboElFotoh, H.M.F., Iyengar, S.S., Chakrabarty, K, "Computing reliability and message delay for Cooperative wireless distributed sensor networks subject to random failures", in IEEE Transactions on Reliability, pp:145 - 155, 2005.

[9] I. F. Akyildiz, W. Su, Y. Sankarasubramaniam, and E. Cayirci, "A Survey on sensor networks", IEEE Communication Magazine, Volume: 40 Issue:8, pp: 102-114, August 2002.

[10] Prasad, D. (2011). A Reliable Security Model Irrespective of Energy Constraints in Wireless Sensor Networks. International Journal of Advanced Computer Science and Applications - IJACSA, 2(4), 20-29.
[11] Jaigirdar, F. T. (2011). Grid Approximation Based Inductive Charger Deployment Technique in Wireless Sensor Networks. International Journal of Advanced Computer Science and Applications - IJACSA, 2(1).

\section{AUTHORS PROFILE}

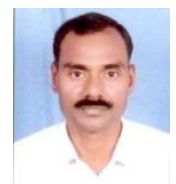

Prof. Vijay Kumar born in Kanpur, India, on $30^{\text {th }}$ June 1972. He received his B.E \& M.E. degrees from Kumaon University Nainital (U.P) and Thapar University Patiala (Punjab) respectively. He has supervised $8 \mathrm{M}$. Tech and $1 \mathrm{M}$. Phil candidates. His research interests are in Wireless Sensor Networks,

Reliability Theory and Artificial Neural Networks, etc. He has about 16 years experience in teaching. He is also a member of IEEE.

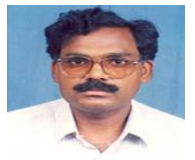

Dr. R. B. Patel received $\mathrm{PhD}$ from IIT Roorkee in Computer Science \& Engineering, PDF from Highest Institute of Education, Science \& Technology (HIEST), Athens, Greece, MS (Software Systems) from BITS Pilani and B. E. in Computer Engineering from M. M. M. Engineering College, Gorakhpur, UP. Dr. Patel is in teaching and Research \& Development since 1991. He has supervised $30 \mathrm{M}$. Tech, $7 \mathrm{M}$. Phil and $2 \mathrm{PhD}$ Thesis. He is currently supervising $3 \mathrm{M}$. Tech, and $8 \mathrm{PhD}$ students. He has published more than 120 research papers in International/National Journals and Refereed International Conferences. He had been awarded for Best Research paper many times in India and abroad. He has written numbers books for engineering courses (These are "Fundamentals of Computing and Programming in C", "Theory of Automata and Formal Languages", "Expert Data Structures with C," "Expert Data Structures with C++," "Art and Craft of C" and "Go Through C". His research interests are in Mobile \& Distributed Computing, Mobile Agent Security and Fault Tolerance, development infrastructure for mobile \& Peer-ToPeer computing, Device and Computation Management, Cluster Computing, Sensor Networks, etc.

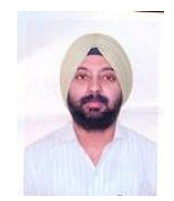

Dr. Manpreet Singh is working as Professor. \& Head of computer science and Engineering department at MMEC, M. M. University Mullana, Ambala, India. He obtained his Ph.D. (Computer Science) from Kurukshetra University. $\mathrm{He}$ has number of publications in International journals/Conferences to his credit. His current research interest includes Grid Computing, Wireless communications, MANETs etc.

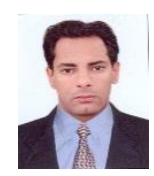

Rohit Vaid received his M. Tech. degree from Maharishi Markandeshwar University Mullana, Ambala, Haryana (India) respectively. He has number of publications in International journals/Conferences to his credit. $\mathrm{He}$ is currently supervising $6 \mathrm{M}$. Tech students. His research interests are in Mobile \& Distributed Computing, Mobile Agent Security and Fault Tolerance, Cluster Computing, Wireless Sensor Networks, etc. 